\title{
Supervision: a Crucial Element of Psychiatric Education
}

\section{Supervision in Psychiatric Practice: Practical Approaches Across Venues and Providers. Edited by Sallie G. De Golia and Kathleen M. Corcoran; American Psychiatric Association Publishing; Washington, DC; 2019; ISBN 978-1-61537-164-8; pp. 457; $\$ 75$ (paperback)}

\author{
Richard Balon $^{1}$ (D)
}

Received: 20 May 2019 / Revised: 20 May 2019 / Accepted: 27 May 2019 / Published online: 4 June 2019

(C) Academic Psychiatry 2019

Supervision is one of the cornerstones of psychiatric education. As one of the editors of Supervision in Psychiatric Practice: Practical Approaches Across Venues and Providers, Sallie De Golia writes, "It is how we learn, it is how we prepare for unsupervised practice, it is how we develop into who we are as professionals. It is also what many of us do as part of our jobs as psychiatrists, psychologists, or other mental health professionals - from supervising psychotherapy to supervising a team of mental health care providers" (p. 3). Yet, as De Golia points out, we receive little or no training in how to supervise, and we cannot even agree on the definition of supervision. That is a bit of a strange reality - we were all supervised during our training, and many of us supervise trainees and/or colleagues, yet we are not clear about the definition, process, structure, and conduct of what we call supervision.

A myriad of questions comes to one's mind when thinking about supervision. We may remember what we, at that time, considered good or bad supervision, but was it really good or bad? Did it really provide what was good and right for us at that time? Did we go through any process of learning to supervise? If we did not, is it possible that we perpetuate some of the mistakes of our supervisors? How much do we know about the area we supervise? How do we know whether we are good at supervising? How can we improve our supervisory skills? What do supervisees think about us as supervisors? How does the program/system view us as supervisors? What should be done if supervision gets stalled? How do we respond to unprofessional behavior of a supervisee?

Richard Balon

rbalon@wayne.edu

1 Wayne State University, Detroit, MI, USA
Supervision in Psychiatric Practice intends to help us answer all of these and many other questions and issues related to supervision. The book has eight parts: introduction, supervision formats, supervision techniques, clinical supervision venues, nonclinical supervision venues, special issues in supervision, legal and ethical issues, and professional development.

The introductory chapter on elements of supervision by De Golia is probably the most useful and thought-provoking text for both novice and practicing supervisors. It points out that one should review adult learning principles, know the context of supervision, anticipate supervisor roles and limitations, establish a working alliance with the supervisee, set the frame, establish learning objectives, and decide about the process of evaluation and providing feedback before starting supervision. (I bet most of us either do not consider all these aspects or consider them instinctively, without much formal deliberation.) De Golia reminds us that "supervisory activities may not always be driven by education or patient safety considerations; they may also be driven by rules and regulations not clearly specified" (p. 7), with the examples of Medicare rules and insurance guidelines given. Regarding supervisory roles, one should realize that these could range from managerial to educational to service to support, and frequently those could overlap. We should also be cognizant of further supervision complexity, because "Supervision is not mentorship, and it is not therapy. It has an evaluative function. However, it may embody aspects of both mentorship and therapy. The supervisor must balance allowing the supervisee to explore internal feelings and reactions with avoiding too much disclosure.... On the other hand, self-disclosure by a supervisor may be quite useful and appropriate, particularly as it relates to supervisees presenting concerns..., whereas this would be more carefully restricted within a therapy context.... Furthermore, though a sound working alliance is critical, the supervisory relationship must tolerate the fact it is not entirely confidential" (p. 8). 
As far as setting the frame, De Golia reminds us that many supervisees, especially junior ones, do not know what to expect from supervision and are not aware of the limits of confidentiality. The last part of this chapter deals with feedback provided to supervisees and includes many important caveats about how to provide it. Finally, De Golia summarizes the role of supervisors: "Supervisors prepare trainees for unsupervised practice, help deepen and enrich the future work of posttrainees, and contribute to supervisees' career development. The process involves a complex set of competencies and approaches that may vary depending on the setting and format of supervision" (pp. 20, 21).

The next 49 brief and narrow-in-scope chapters summarize almost every corner of supervisory activity. The chapters are well organized and include specific challenges and strategies for supervision in particular situations or settings, questions for supervisors, additional resources, and solid references.

I will note a few examples to illustrate the degree of thoroughness of this volume. Some chapters bring the reader's attention to important aspects of behavior to be avoided, for example, a tendency toward disinhibition in videoconferences and email interactions. I was not sure why some chapters were included (e.g., the chapter on cotherapy), and some chapters covered too little (e.g., the chapter about night float might also have discussed the stressful aspects of such a coverage arrangement). The chapter on psychodynamic supervision describes a typical supervisory hour, which is very helpful. I liked the thorough and very useful discussion of ethics of child and adolescent psychiatry supervision, including the issues of assent and informed consent. The chapter on leadership supervision notes an old adage we should include in our deliberations: "Good judgment comes from experience, experience comes from bad judgment." Very helpful are chapters on how to select an individual psychotherapy supervisor and on how supervisees can make the most of supervision, or how consulting differs from supervising.

The chapter on unprofessional behavior includes a very important description of unprofessional behaviors and their management:

Mild problems in professionalism could include supervisees who are late to supervision, refer to patients in disrespectful manner, or who do not wear appropriate clinical attire. Mild problems may be reclassified as moderate if the behaviors escalate in frequency or severity, or the supervisees are unresponsive to feedback given (e.g., supervisees are routinely late or miss supervision following feedback on these behaviors). Examples of moderate professionalism problems include inadequate documentation of clinical encounters or failure to seek supervision when appropriate. Severe violations of professionalism may include illegal (e.g., stealing property) or unethical (e.g., having sex with a patient) behaviors and may require immediate action, including remediation, probation, or termination." (pp. 324, 325)

The text emphasizes the need to deal with these behaviors resolutely and that remediation or individualized learning plans should be tailored and addressed through a behavioral approach. Similarly revealing is the chapter on ethical and legal considerations in supervision, especially the explanation of vicarious and direct liability.

Supervision in Psychiatric Practice is a well put-together, comprehensive resource book and a very good start to finally seriously approaching an important part of psychiatric education and training. It has very few weaknesses. For example, I would have liked to see a thorough discussion of supervision of combining psychopharmacology and psychotherapy, whether in a collaborative or split setting (e.g., supervising psychiatry residents and psychology interns who share patients) or a stronger endorsement of the need to develop training programs for supervisors (I think psychologists are much ahead of psychiatrists with this part of supervisor development). As noted in the final chapter (almost hidden at the end, instead of being one of the beginning chapters), "Clinical supervision is understudied, and there is even less literature regarding professional development programs for psychiatry supervisors" (p. 409).

Nevertheless, those are minor points that do not detract from the enormous value of this volume. The book should be read by every program director and every supervisor. It will help us prepare generations of more competent supervisors and more competent trainees.

\section{Compliance with Ethical Standards}

Disclosure Dr. Balon is a member of the APA Publishing Editorial Board and receives royalties from books he has authored and edited.

Publisher's Note Springer Nature remains neutral with regard to jurisdictional claims in published maps and institutional affiliations. 